PROCEEDINGS OF THE

AMERICAN MATHEMATICAL SOCIETY

Volume 132, Number 6, Pages 1869-1872

S 0002-9939(03)07298-8

Article electronically published on November 21, 2003

\title{
NOTE ON QUANTUM UNIQUE ERGODICITY
}

\author{
STEVE ZELDITCH
}

(Communicated by Christopher D. Sogge)

\begin{abstract}
We prove that (near) off-diagonal matrix elements $\left\langle A \varphi_{i}, \varphi_{j}\right\rangle(i \neq$ $j$ ) of pseudodifferential operators relative to eigenfunctions of quantum uniquely ergodic Laplacians vanish as the eigenvalues tend to infinity. It follows that QUE systems cannot have quasi-modes with singular limits and a bounded number of essential frequencies, as is believed to occur for the stadium and other examples.
\end{abstract}

The purpose of this note is to record an observation about quantum unique ergodicity (QUE) which is relevant to the recent construction of H. Donnelly $[\mathrm{D}$ of quasi-modes on certain non-positively curved surfaces, and to similar quasi-mode constructions known for many years as bouncing ball modes on Bunimovich stadia (BSS], [H] , BZ1], BZ2]). Our new observation (Proposition 0.1) is the asymptotic vanishing of near off-diagonal matrix elements for eigenfunctions of QUE systems. As a corollary, we find that quantum ergodic (QE) systems possessing quasi-modes with singular limits and with a limited number of frequencies cannot be QUE.

We begin by recalling that QUE (for Laplacians) concerns the matrix elements $\left\langle A \varphi_{i}, \varphi_{j}\right\rangle$ of pseudodifferential operators relative to an orthonormal basis $\left\{\varphi_{j}\right\}$ of eigenfunctions

$$
\Delta \varphi_{j}=\lambda_{j}^{2} \varphi_{j}, \quad\left\langle\varphi_{j}, \varphi_{k}\right\rangle=0
$$

of the Laplacian $\Delta$ of a compact Riemannian manifold $(M, g)$. We denote the spectrum of $\Delta$ by $S p(\Delta)$. By definition, $\Delta$ is QUE if

$$
\left\langle A \varphi_{j}, \varphi_{j}\right\rangle \rightarrow \int_{S^{*} M} \sigma_{A} d L
$$

where $d L$ is the (normalized) Liouville measure on the unit (co-)tangent bundle. The term "unique" indicates that no subsequence of density zero of eigenfunctions need be removed when taking the limit.

The main result of this note is that all off-diagonal terms of QUE systems tend to zero if the eigenvalue gaps tend to zero. This strengthens the conclusion of $[\mathbf{Z}]$ that almost all off-diagonal terms (with vanishing gaps) tend to zero in general QE situations. As will be seen below, it also provides evidence that Donnelly's examples are non-QUE and establishes a localization statement of Heller and O'Connor [HO].

Received by the editors January 28, 2003 and, in revised form, March 10, 2003.

2000 Mathematics Subject Classification. Primary 58J50, 58J40, 35P99, 81S10.

This research was partially supported by NSF grant DMS-0071358 and by the Clay Mathematics Institute.

(C)2003 American Mathematical Society 
Proposition 0.1. Suppose that $\Delta$ is QUE. Suppose that $\left\{\left(\lambda_{i_{r}}, \lambda_{j_{r}}\right), i_{r} \neq j_{r}\right\}$ is a sequence of pairs of eigenvalues of $\sqrt{\Delta}$ such that $\lambda_{i_{r}}-\lambda_{j_{r}} \rightarrow 0$ as $r \rightarrow \infty$. Then $d \Phi_{i_{r}, j_{r}} \rightarrow 0$, where we define the distributions $d \Phi_{i, j} \in \mathcal{D}^{\prime}\left(S^{*} M\right)$ by

$$
\left\langle O p(a) \varphi_{i}, \varphi_{j}\right\rangle=\int_{S^{*} M} a d \Phi_{i, j}
$$

where $a \in C^{\infty}\left(S^{*} M\right)$.

Proof. Let $\left\{\lambda_{i_{r}}, \lambda_{j_{r}}\right\}$ be any sequence of pairs with the gap $\lambda_{i_{r}}-\lambda_{j_{r}} \rightarrow 0$. It is then known that any weak* limit $d \nu$ of the sequence $\left\{d \Phi_{i_{r}, j_{r}}\right\}$ is a measure invariant under the geodesic flow $[\mathrm{Z},[\mathrm{D}]$. The weak limit is defined by the property that

$$
\left\langle A^{*} A \varphi_{i_{r}}, \varphi_{j_{r}}\right\rangle \rightarrow \int_{S^{*} M}\left|\sigma_{A}\right|^{2} d \nu .
$$

If the eigenfunctions are real, then $d \nu$ is a real (signed) measure.

Our first observation is that any such weak limit must be a constant multiple of Liouville measure $d L$. Indeed, we first have

$$
\left|\left\langle A^{*} A \varphi_{i}, \varphi_{j}\right\rangle\right| \leq\left|\left\langle A^{*} A \varphi_{i}, \varphi_{i}\right\rangle\right|^{1 / 2}\left|\left\langle A^{*} A \varphi_{j}, \varphi_{j}\right\rangle\right|^{1 / 2} .
$$

Taking the limit along the sequence of pairs, we obtain

$$
\left.\left.\left|\int_{S^{*} M}\right| \sigma_{A}\right|^{2} d \nu\left|\leq \int_{S^{*} M}\right| \sigma_{A}\right|^{2} d L .
$$

It follows that $d \nu<<d L$ (absolutely continuous). But $d L$ is an ergodic measure. So if $d \nu=f d L$ is an invariant measure with $f \in L^{1}(d L)$, then $f$ is constant. Thus,

$$
d \nu=C d L, \text { for some constant } C .
$$

We now observe that $C=0$ if $\varphi_{i} \perp \varphi_{j}$ (i.e., if $i \neq j$ ). This follows if we substitute $A=I$ in (2), and use orthogonality and (5).

This result has implications for the possible "scarring" of quasi-modes of QUE systems. We first recall that a quasi-mode of order $s$ for $\Delta$ is a sequence $\left\{\psi_{k}\right\}$ of $L^{2}$-normalized functions that solves

$$
\left\|\left(\Delta-\mu_{k}\right) \psi_{k}\right\|_{L^{2}}=O\left(\mu_{k}^{-s / 2}\right),
$$

for a sequence of quasi-eigenvalues $\mu_{k}$ (see [CdV] for background). In particular, a quasi-mode of order 0 satisfies $\left\|\left(\Delta-\mu_{k}\right) \psi_{k}\right\|_{L^{2}}=O(1)$. Such (relatively low-order) quasi-modes can be easily constructed for the stadium (BSS], [H], BZ1, [BZ2] and for Donnelly's surfaces $[\mathrm{D}]$. As with eigenfunctions, we can consider the limits

$$
\left\langle A \psi_{j}, \psi_{j}\right\rangle \rightarrow \int_{S^{*} M} \sigma_{A} d \nu
$$

of diagonal matrix elements $\left\langle A \psi_{j}, \psi_{j}\right\rangle$ of quasi-modes. We say that the quasi-mode "scars" if the limit measure $d \nu$ has a nonzero singular component relative to $d L$. For instance, bouncing ball modes of stadia "scar" on the Lagrangian manifold with boundary formed by the bouncing ball orbits in the central rectangle, and the similar quasi-modes in [D] scar on the circles in the cylindrical part. The existence of such scarring quasi-modes suggests that these systems are not QUE.

To explore this suggestion, we consider the decomposition of scarring quasimodes into sums of true eigenfunctions. 
Definition 1. We say that a quasi-mode $\left\{\psi_{k}\right\}$ of order 0 as in (6) with $\left\|\psi_{k}\right\|_{L^{2}}=1$ has $n(k)$ essential frequencies if, for each $k$, there exists $\delta>0$ and a subset $\Lambda_{k} \subset$ $S p(\Delta) \cap\left[\mu_{k}-\delta, \mu_{k}+\delta\right]$ with $n(k)=\# \Lambda_{k}$, and constants $c_{k j} \in \mathbb{C}$, such that

$$
\psi_{k}=\sum_{j: \lambda_{j}^{2} \in \Lambda_{k}} c_{k j} \varphi_{j}+\eta_{k}, \text { with }\left\|\eta_{k}\right\|_{L^{2}}=o(1) .
$$

The following problems then seem interesting (the first is implicit in [HO]).

- Bound the number $n(k)$ of essential frequencies of a quasi-mode $\left\{\psi_{k}\right\}$ of order 0 that tends to a singular (i.e., non-Liouville) classical limit, e.g., a periodic orbit measure.

- Bound the order $s$ of a quasi-mode with a singular limit (intuitively, the diameter of the set of eigenvalues that composes its packet of eigenfunctions.)

In other words, the questions are whether one can build a quasi-mode with a singular limit and (i) with anomalously few essential frequencies, or (ii) with anomalously low order. This softens the mathematicians' criterion of scarring as the existence of a sequence of actual modes (eigenfunctions) whose limit measure $\nu$ in (2) has a singular component relative to Liouville measure $\underline{\mathrm{S}}$.

The following shows that quasi-modes with a uniformly bounded number of essential frequencies and singular limits do not exist for QUE systems.

Corollary 0.2. If there exists a quasi-mode $\left\{\psi_{k}\right\}$ of order 0 for $\Delta$ as in (8) and a constant $C>0$ with the properties:

(i) $n(k) \leq C, \forall k$,

(ii) $\left\langle A \psi_{k}, \psi_{k}\right\rangle \rightarrow \int_{S^{*} M} \sigma_{A} d \mu$ where $d \mu \neq d L$,

then $\Delta$ is not QUE.

Proof. We argue by contradiction. If $\Delta$ were QUE, we would have (in the notation of (8)):

$$
\begin{aligned}
\left\langle A \psi_{k}, \psi_{k}\right\rangle & =\sum_{i, j: \lambda_{i}^{2}, \lambda_{j}^{2} \in \Lambda_{k}} c_{k j} \bar{c}_{k i}\left\langle A \varphi_{i}, \varphi_{j}\right\rangle+o(1) \\
& =\sum_{j: \lambda_{j}^{2} \in \Lambda_{k}}\left|c_{k j}\right|^{2}\left\langle A \varphi_{j}, \varphi_{j}\right\rangle+\sum_{i \neq j: \lambda_{i}^{2}, \lambda_{j}^{2} \in \Lambda_{k}} c_{k j} \bar{c}_{k i}\left\langle A \varphi_{i}, \varphi_{j}\right\rangle+o(1) \\
& =\int_{S^{*} M} \sigma_{A} d L+o(1),
\end{aligned}
$$

by Proposition 0.1. This contradicts (ii). In the last line, we used that $\left|\lambda_{i}-\lambda_{j}\right| \rightarrow 0$ if $\lambda_{i}^{2}, \lambda_{j}^{2} \in \Lambda_{k}$ and that $\sum_{j: \lambda_{j}^{2} \in \Lambda_{k}}\left|c_{k j}\right|^{2}=1+o(1)$, since $\left\|\psi_{k}\right\|_{L^{2}}=1$.

The assumption that $n(k) \leq C$ could be weakened if we knew something about the rate of decay of the individual elements $\left\langle A \varphi_{j}, \varphi_{k}\right\rangle$ and $\left|\left\langle A \varphi_{j}, \varphi_{j}\right\rangle-\int_{S^{*} M} \sigma_{A} d L\right|$.

We now consider the implications for the stadium and for Donnelly's surface. In both cases, it is unknown how many essential frequencies are needed to build the associated bouncing ball quasi-modes. On average, intervals of fixed width have a uniformly bounded number of $\Delta$-eigenvalues in dimension 2 , and this suggests that $n(k) \leq C$. Our result would then show that such systems are automatically not QUE (as is widely believed). On the other hand, the standard remainder estimate for Weyl's law allows $O(\sqrt{k})$ eigenvalues of $\Delta$ in the interval $\left[\mu_{k}-\delta, \mu_{k}+\delta\right]$, and it is possible that exceptionally high clustering occurs around the bouncing ball quasi-eigenvalues. Numerical evidence $([\mathrm{H}],[\mathrm{HO},[\mathrm{BSS}])$ seems to show that no 
exceptional clustering occurs and that bouncing ball quasi-modes are combinations of a fixed number of actual modes. But there are at this time no rigorous results of this kind or in general on the soft scarring criteria above.

We close with some recent references to the literature on eigenfunction scarring. At this time, no $\Delta$ are proved to be QUE and none are proved to be non-QUE. But very recently E. Lindenstrauss $[\mathrm{L}]$ has proved that the special basis of Hecke eigenfunctions of arithmetic hyperbolic surfaces has the QUE property (see also $[\mathrm{S}],[\mathrm{RS}], \mathrm{BL},[\mathrm{W}]$ for prior results in this line). Also, Faure, Nonnenmacher, and de Bievre have recently proved that certain quantum cat maps are not QUE [FN], [FND]. Burq and Zworski [BZ1], BZ2 have recently given upper bounds on the concentration of eigenfunctions in the central part of stadia or in collars around hyperbolic closed geodesics Riemannian manifolds, which show that the optimal order of quasi-modes with singular concentration in these regions is 0 . They further show that stadium eigenfunctions cannot scar on smaller sets than the entire set of bouncing ball orbits.

\section{REFERENCES}

[BSS] A. Backer, R. Schubert, and P. Stifter, On the number of bouncing ball modes in billiards, J. Phys. A 30 (1997), no. 19, 6783-6795.

[BL] J. Bourgain and E. Lindenstrauss, Entropy of Quantum Limits, Commun. Math. Phys. 233 (2003), 153-171.

[BZ1] N. Burq and M. Zworski, Control in the presence of a black box, arxiv preprint math.AP/0304184 (2003).

[BZ2] N. Burq and M. Zworski, Bouncing ball modes and quantum chaos, arxiv preprint math.AP/0306278 (2003).

[CdV] Y. Colin de Verdière, Quasi-modes sur les variétés Riemanniennes, Invent. Math. 43 (1977), no. 1, 15-52. MR 58:18615

[D] H. G. Donnelly, Quantum unique ergodicity, Proc. Amer. Math. Soc. 131 (2003), no. 9, 2945-2951.

[FN] F. Faure and S. Nonnenmacher, On the maximal scarring for quantum cat map eigenstates, arxiv preprint nlin.CD/0304031 (2003).

[FND] F. Faure, S. Nonnenmacher, and S. De Bievre, Scarred eigenstates for quantum cat maps of minimal periods, arxiv preprint nlin.CD/0207060 (2003), Comm. Math. Phys. 239 (2003), 449-492.

$[\mathrm{H}] \quad$ E. J. Heller, Wavepacket dynamics and quantum chaology. Chaos et physique quantique (Les Houches, 1989), 547-664, North-Holland, Amsterdam, 1991. MR 94i:81031

[HO] E. J. Heller and P. W. O'Connor, Quantum localization for a strongly classically chaotic system, Phys. Rev. Lett. 61 (20) (1988), 2288-2291. MR 89j:81069

[L] E. Lindenstrauss, Invariant measures and arithmetic quantum unique ergodicity, preprint, 2003.

[RS] Z. Rudnick and P. Sarnak, The behaviour of eigenstates of arithmetic hyperbolic manifolds, Comm. Math. Phys. 161 (1994), no. 1, 195-213. MR 95m:11052

[S] P. Sarnak, Arithmetic quantum chaos, The Schur lectures (1992) (Tel Aviv), 183-236, Israel Math. Conf. Proc., 8, Bar-Ilan Univ., Ramat Gan, 1995. MR 96d:11059

[W] S. A. Wolpert, The modulus of continuity for $\Gamma_{0}(m) \backslash \mathbb{H}$ semi-classical limits, Comm. Math. Phys. 216 (2001), no. 2, 313-323. MR 2002f:11059

[Z] S. Zelditch, Quantum transition amplitudes for ergodic and for completely integrable systems, J. Funct. Anal. 94 (1990), no. 2, 415-436. MR 92b:58181

Department of Mathematics, Johns Hopkins University, Baltimore, Maryland 21218

E-mail address: zelditch@math.jhu.edu 